\title{
Toward a Deeper Understanding of the Role of Interaction in Information Visualization
}

\author{
Ji Soo Yi, Youn ah Kang, John T. Stasko, Member, IEEE, and Julie A. Jacko
}

\begin{abstract}
Even though interaction is an important part of information visualization (Infovis), it has garnered a relatively low level of attention from the Infovis community. A few frameworks and taxonomies of Infovis interaction techniques exist, but they typically focus on low-level operations and do not address the variety of benefits interaction provides. After conducting an extensive review of Infovis systems and their interactive capabilities, we propose seven general categories of interaction techniques widely used in Infovis: 1) Select, 2) Explore, 3) Reconfigure, 4) Encode, 5) Abstract/Elaborate, 6) Filter, and 7) Connect. These categories are organized around a user's intent while interacting with a system rather than the low-level interaction techniques provided by a system. The categories can act as a framework to help discuss and evaluate interaction techniques and hopefully lay an initial foundation toward a deeper understanding and a science of interaction.
\end{abstract}

Index Terms - Information visualization, interaction, interaction techniques, taxonomy, visual analytics

\section{INTRODUCTION}

Information visualization (Infovis) systems, at their core, appear to have two main components: representation and interaction. The representation component, whose roots lie in the field of computer graphics, concerns the mapping from data to representation and how that representation is rendered on the display. The interaction component involves the dialog between the user and the system as the user explores the data set to uncover insights. The interaction component's roots lie in the area of human-computer interaction (HCI). Although discussed as two separate components, representation and interaction clearly are not mutually exclusive. For instance, interaction with a system may activate a change in representation. Nonetheless, the two components seem to compose the two fundamental aspects of Infovis systems, and it seems reasonable to consider what each contributes to an end-user's experience.

We argue that the representation component has received the vast majority of attention in Infovis research. A cursory scan of a recent conference proceedings or journal issues in the area will uncover many articles about new representations of data sets, but interaction is often relegated to a secondary role in these articles. Interaction rarely is the main focus of research efforts in the field, essentially making it the "little brother" of Infovis. In other words, it is overshadowed by the more noteworthy representation aspects. A few papers have mainly focused on the interactive aspects of Infovis (e.g., $[10,15,25,47])$, but these are relatively uncommon when compared to papers introducing new data representations.

Interaction is an essential part of Infovis, however. Without interaction, an Infovis technique or system becomes a static image or autonomously animated images (e.g., InfoCanvas [28]). While static images clearly have analytic and expressive value (e.g., [8, 29, 46]), their usefulness becomes more limited as the data set that they represent grows larger with more variables. Actually, even with a static image such as a poster, a user (or a reader) will often perform several interactions (e.g., rotating the poster, looking closer/further,

- Ji Soo Yi is with Health Systems Institute \& H. Milton Stewart School of Industrial and Systems Engineering, Georgia Institute of Technology, EMail: jisoo.yi@hsi.gatech.edu.

- Youn ah Kang and John T. Stasko are with School of Interactive Computing \& GVU Center, Georgia Institute of Technology, E-Mail: ykang3@mail.gatech.edu and stasko@cc.gatech.edu.

- Julie A. Jacko is with Health Systems Institute, The Wallace H. Coulter Department of Biomedical Engineering, \& College of Computing, Georgia Institute of Technology \& Emory University, E-Mail: jacko@hsi.gatech.edu

Manuscript received 31 March 2007; accepted 1 August 2007; posted online 27 October 2007.

For information on obtaining reprints of this article, please send e-mail to: tvcg@computer.org. and jotting down notes on the poster). Spence even suggests the notion of "passive interaction" through which the user's mental model on the data set is changed or enhanced [38]. Finally, through interaction, some limits of a representation can be overcome, and the cognition of a user can be further amplified (e.g., [15, 29]).

The importance of interaction and the need for its further study seem undisputed. For example, the recent book Illuminating the Path: The Research and Development Agenda for Visual Analytics calls for further research on interaction:

\begin{abstract}
"Recommendation 3.3: Create a new science of interaction to support visual analytics. The grand challenge of interaction is to develop a taxonomy to describe the design space of interaction techniques that supports the science of analytic reasoning. We must characterize this design space and identify under-explored areas that are relevant to visual analytics. Then, $R \& D$ should be focused on expanding the repertoire of interaction techniques that can fill those gaps in the design space." ([45], p. 76)
\end{abstract}

This recommendation concerns visual analytics which is not equivalent to Infovis, but the two clearly share much in common and the motivation for this call can equally be applied to Infovis.

While we believe that few would argue with the merits of the goals in the recommendation, precisely defining what is being called for is not so easy. What does it mean to create a "science of interaction" in visual analytics and Infovis? The recommendation speaks of developing a taxonomy of interaction techniques and identifying under-explored areas for future research. These are noble efforts, but we believe that a science of interaction also should involve gaining a deeper understanding of the utility and value of interaction in these fields. What does interaction contribute to the analytic process?

For that matter, we might raise questions about the nature of interaction itself. In the context of Infovis, what is interaction and interactive behavior? Operations such as moving a dynamic query slider [3] to narrow the set of data points being shown or selecting an alternate point in a fisheye view [19] to change the focus seem like clear examples of interactive behavior. But consider a system where the user selects a menu operation to change from a scatter plot to a parallel coordinates of the data. Is that interaction?

The purpose of this article relates to the recommendation from Illuminating the Path that was discussed above. Defining a science of interaction is a lofty goal and we do not purport to do so here, but we do seek to take some initial steps toward that goal. Our objective is to further current understandings of the role that interaction plays 
in Infovis. More specifically, we seek to identify the fundamental ways that interaction is used in Infovis systems and the benefits it provides to them (and to users).

In the next section, we review prior research on interaction in Infovis and examine how other researchers have defined and characterized its virtues. In Section 3, we describe the research methods used to survey and analyze these interaction techniques. In Section 4, we describe the results of an extensive analytic investigation of interaction techniques and introduce seven fundamental ways that interaction contributes to the explorations and analyses people perform while using Infovis systems.

\section{BACKGROUND}

It seems appropriate to start a discussion of interaction in Infovis with a definition of the term, but in fact, finding a solid definition of interaction is challenging. In the broader context of HCI, Dix et al. simply describe interaction as "the communication between user and the system" (p. 124) [16]. Becker, Cleveland, and Wilks compactly define interaction as direction manipulation and instantaneous change [6]. Since interaction can occur even with a static image as described previously [38], interaction is certainly not a tangible concept. That is likely why Beaudouin-Lafon mentions that "HCI research is far from having solid (and falsifiable) theories of interaction" (p. 16) [5].

Nonetheless, interaction techniques are less difficult to define and are more tangible concepts than interaction itself. A static image does not have an associated interaction technique even though users can interact with it. Foley et al. define an interaction technique as a way of using a physical input/output device to perform a generic task in a human-computer dialogue [18].

The definition of interaction techniques in the context of Infovis should extend Foley's definition, however, which was grounded in the general context of HCI. As Ware identifies via the phrase, "asymmetry in data rates" (p.382) [51], the amount of data flowing from Infovis systems to users is far greater than from users to systems. Thus, interaction techniques in Infovis seem more designed for changing and adjusting visual representation than for entering data into systems, which clearly is an important aspect of interaction in HCI.

We view interaction techniques in Infovis as the features that provide users with the ability to directly or indirectly manipulate and interpret representations. According to this view, a static image or an autonomously animated representation does not have associated interaction techniques. However, a menu interface for changing from a scatter plot to a parallel coordinates view is an interaction technique since it allows users to manipulate a representation even though it may be less interactive or direct. (Here and throughout this paper, we intentionally use the term "user" rather than "viewer" or "people" to emphasize the fact that users actively use and interact with Infovis systems.)

Taxonomies of interaction techniques would be helpful to achieve a better understanding of the design space of interaction. Table 1 summarizes several studies in Infovis proposing taxonomies that we think are relevant to the examination of interaction techniques. Even though many of the studies share common units, the taxonomies have significantly different levels of granularity. Some try to categorize low-level interaction techniques (e.g., [9, 12, 15, 24, 37, 54]); some provide dimensions to describe interaction techniques (e.g., [38, 47]); another moves past the low-level interaction techniques to provide a broader view of interaction including notions such as interaction spaces and parameters (e.g., [50]); while others focus more on users' tasks (e.g., $[4,56])$. This divergence suggests that there may be multiple ways or granularities to describe interaction techniques, which is also in line with Norman's action cycle [30] that describes interaction between a user and the world using multiple steps (i.e., forming the goal, forming the intention, specifying an action, executing the action, perceiving the state of the world, interpreting the state of the world, and evaluating the outcome). Also, this divergence implies that defining a comprehensive taxonomy is challenging. Since Infovis is still a growing field, it is highly possible that an interaction technique developed in the future will not be clearly categorized by one of the low-level interaction technique taxonomies.

Table 1. Infovis Taxonomies Relevant to Interaction Techniques

Publication

Taxonomic units

Taxonomies of low-level interaction techniques

Shneiderman (1996) Overview, zoom, filter, details-on-demand, relate, [37] history, and extract

Buja, Cook, and Focusing (choice of [projection, aspect ratio,

Swayne (1996) [9] zoom, pan], choice of [variable, order, scale, scaleaspect ratio, animation, and 3-D rotation]), linking (brushing as conditioning / sectioning / database query), and arranging views (scatter plot matrix and conditional plot)

Chuah and Roth (1996) Basic visualization interaction (BVI) operations: [13] graphical operations (encode data, set graphical value, manipulate objects), set operations (create set, delete set, summarize set, other), and data operations (add, delete, derived attributes, other)

Dix and Ellis (1998) Highlighting and focus, accessing extra

[15] information - drill down and hyperlinks, overview and context, same representation / changing parameters, same data / changing representation, linking representation - temporal fusion

Keim (2002) [24] Dynamic projections, interactive filtering, interactive zooming, interactive distortion, interactive linking and brushing

Wilkinson (2005) [54] Filtering (categorical/continuous/multiple/fast filtering), navigating (zooming/panning/lens), manipulating (node dragging/categorical reordering), brushing and linking (brush shapes/brush logic/fast brushing), animating (frame animation), rotating, transforming (specification/assembly/display/tap/2 taps/3 taps) Taxonomical dimensions of interaction techniques

Tweedie (1997) [47] Interaction types (manual, mechanized, instructable, steerable, and automatic) and directness (direct and indirect manipulation)

Spence (2007) [38] Interaction modes (continuous, stepped, passive, and composite interaction)

A taxonomy of interaction operations

Ward and Yang (2004) interaction operators (navigation, selection,

[50] distortion), interaction spaces (screen-space, data value-spaces, data structure-space, attribute-space, object-space, and visualization structure-space), and interaction parameters (focus, extents, transformation, and blender)

Taxonomies of user tasks

Zhou and Feiner Relational visual tasks (associate, background,

(1998) [56] categorize, cluster, compare, correlate, distinguish, emphasize, generalize, identify, locate, rank, reveal, switch) and direct visual organizing and encoding tasks (encode)

Amar, Eagan, and Retrieve value, filter, compute derived value, find Stasko (2005) [4] extremum, sort, determine range, characterize distribution, find anomalies, cluster, and correlate

While these taxonomies are certainly useful for better understanding interaction, to us they still lack something important. The first three sets focus strongly on interaction techniques and are relatively system-centric. The last set focuses on user goals without a main focus on interaction. We believe it would be beneficial to bridge these two efforts - to connect user objectives with the interaction techniques that help accomplish them.

Finally, measuring the effectiveness of a taxonomy is difficult itself. We are drawn to a discussion of this issue by BeaudouinLafon [5] who proposes three dimensions to evaluate interaction models: 1) descriptive power, "the ability to describe a significant 
range of existing interface"; 2) evaluative power: "the ability to help assess multiple design alternatives"; and 3) generative power: "the ability to help designers create new designs" (p. 17). None of the taxonomies listed above appear to provide all three levels.

\section{Methods}

In order to more systematically understand the underlying mechanisms of interaction, we began this research with the goal of building a comprehensive list of Infovis interaction techniques. Since it clearly would not be possible to examine all existing systems and techniques, we decided instead to review existing literature and Infovis systems as follows.

We began by reviewing existing literature containing taxonomies of Infovis interaction techniques, as mentioned in the Background section just above. Next, we examined a number of commercial Infovis systems (e.g., SeeIT by ADVISOR Solutions, Inc. (formerly Visual Insights) [1], Spotfire ${ }^{\circledR}$ by Spotfire, Inc. [2, 41], TableLens ${ }^{\mathrm{TM}}$ by Inxight Software, Inc. [23, 33], and InfoZoom ${ }^{\circledR}$ by humanIT [22, 40]) since they, as general purpose Infovis tools, tend to have a broad set of multiple interaction techniques. We also reviewed articles introducing new Infovis interaction techniques (e.g., pan \& zoom, overview \& details, focus + context, and filter). Finally, we selected well-known papers in sub-areas of Infovis (e.g., multivariate, timeseries, hierarchical, software, security, geographic, and social visualization) to cover various application areas. In total, we surveyed 59 papers and 51 systems and collected 311 individual interaction techniques actually implemented in Infovis systems.

Even though the list of interaction techniques was growing larger and larger, the efforts left us somewhat unsatisfied. It was not clear how useful this list of techniques would be or more importantly, whether it would be descriptive and meaningful. As the gathering process progressed, however, we began to notice common sets of techniques emerging and some styles of interaction being listed more frequently.

Accordingly, we decided to aggregate and cluster the different techniques by using an affinity diagramming method. We grouped similar interaction techniques and iteratively refined the groups according to the core concepts. During the grouping process, several competing grouping schemes emerged. Initial groups tended to be commonly-used interaction techniques in different Infovis systems, which were not that different from existing taxonomies of low-level interaction techniques. However, we soon found that these grouping schemes could not be robust because there were numerous variants of interaction techniques that did not fall into any commonly used interaction technique. We realized that for different representation techniques, different interaction techniques are used to perform a similar task or achieve a similar goal. For example, suppose that a user is exploring the relationship between two particular variables. In a scatter plot style of visualization as in Spotfire [2], this goal is achieved by designating the two variables to be plotted on the $\mathrm{x}$ and $y$ axes. In TableLens [33], however, the goal can be achieved by positioning the two variables next to each other and sorting values with respect to one of the variables.

Thus, we turned our attention to what users achieve by using the interaction techniques rather than how the techniques provided by Infovis systems work. In doing so, we realized that many different styles of interaction techniques serve a relatively small set of purposes. For example, unfolding sub-categories in an interactive pie chart [15], drill-down in a treemap [36], and semantic zooming [32] all may appear very different, but we argue that they serve the same purpose, getting more details.

After several iterations of clustering the techniques, the notion of aggregating them by the user's intent in performing an interactive operation began to emerge. We found that the concept of "What a user wants to achieve', herein described as "user intent," is quite effective to classify the low-level interaction techniques into a small number of descriptive high-level categories.

\section{Categories}

Based on the notion of user intent, the following seven categories of interaction in Infovis emerged from our study. Each category will be discussed in more detail in a subsequent sub-section. To each category, as a title, we assigned a short identifying name (e.g., Select) and also an illustrative phrase that captures the essence of the user's intent in performing the interaction. We describe each category to provide a definition of what it means and we also include exemplary individual interaction techniques that fall within that category.

- $\quad$ Select: mark something as interesting

- Explore: show me something else

- Reconfigure: show me a different arrangement

- Encode: show me a different representation

- Abstract/Elaborate: show me more or less detail

- Filter: show me something conditionally

- Connect: show me related items

\subsection{Select: mark something as interesting}

Select interaction techniques provide users with the ability to mark a data item(s) of interest to keep track of it. When too many data items are presented on a view, or when representations are changed, it is difficult for users to follow items of interest. By making items of interest visually distinctive, users can easily keep track of them even in a large data set and/or with changes in representations.

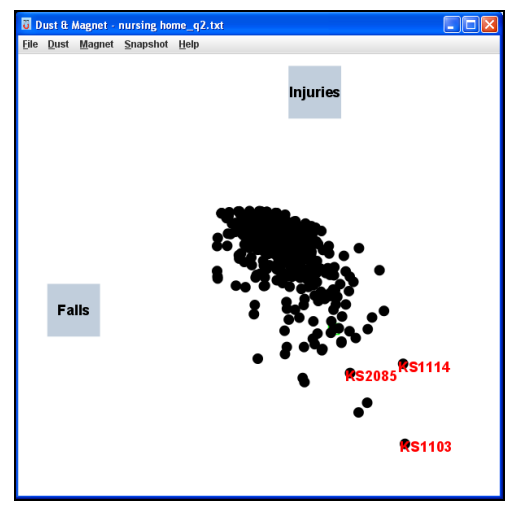

Fig. 1. A screen shot of Dust \& Magnet showing the marking feature.

As shown in Fig. 1, the marking feature in Dust \& Magnet [55], which visualizes data items as specks of iron that move when magnets (attributes) are manipulated, is an example of Select. With this technique, users can mark data items, and the marked items (KS1114, KS2085, and KS1103 in the figure) are labeled in red, so even after rearranging items, users can easily track and identify the location of items of interest. The spotlight feature in TableLens [33], a system that visualizes numerical data using bar charts in a tabular view, is a similar interaction technique except that Spotlight highlights data items instead of labeling. Yet another example of Select is the placemark feature in Google Earth [20], an interactive $3 \mathrm{D}$ geographic visualization tool. By putting a placemark on a location of interest, users can return to the location easily.

Interestingly, Select interaction techniques seem to work as a preceding action to subsequent operations. As shown in the Dust \& Magnet and TableLens examples, users select data items of interest before rearranging, so that they can see where the items of interest would be located in the new arrangement. Rather than acting as a standalone technique, Select interaction is coupled with other interaction techniques to enrich user exploration and discovery.

\subsection{Explore: show me something else}

Explore interaction techniques enable users to examine a different subset of data cases. When users view data using an Infovis system, they often can only see a limited number of data items at a time because of some combination of the large scale of the data set, view 
and/or screen limitations, and fundamental perceptual and cognitive limitations in human information processing. Infovis system users typically examine a subset of the data to gain understanding and insight, and then they move on to view some other data. Explore interactions do not necessarily make complete changes in the data being viewed, however. More frequently, some new data items enter the view as others are removed.

The most common Explore interaction technique in our survey is panning. Panning refers to the movement of a camera across a scene or scene movement while the camera stays still. Panning is often achieved by a special mode where the user grabs the scene and moves it with a mouse or by simply altering the view via scrollbars. Many Infovis systems use panning techniques: for example, Spotfire [2], Vizster [21], Dust \& Magnet [55], and SeeIT [1].

Another example of an Explore interaction is the Direct-Walk technique. Direct-Walk allows users to smoothly move the viewing focus from one position in information structure to another by "a series of mouse points or other direct-manipulation methods" (p. 239) [11]. The hyperlink feature in the Jazz zooming interface toolkit [7] is an example of Direct-Walk. Hyperlinks move the user from one point in the information space to another in a smooth, animated transition. An online graphical dictionary, Visual Thesaurus ${ }^{\circledR}$ [44], is another example of Direct-Walk. In Visual Thesaurus ${ }^{\circledR}$, a searched vocabulary is displayed at the center surrounded by related vocabularies as shown Fig. 2. When one of surrounding words is clicked, the word smoothly comes to the center and new related words surround this newly centered word.

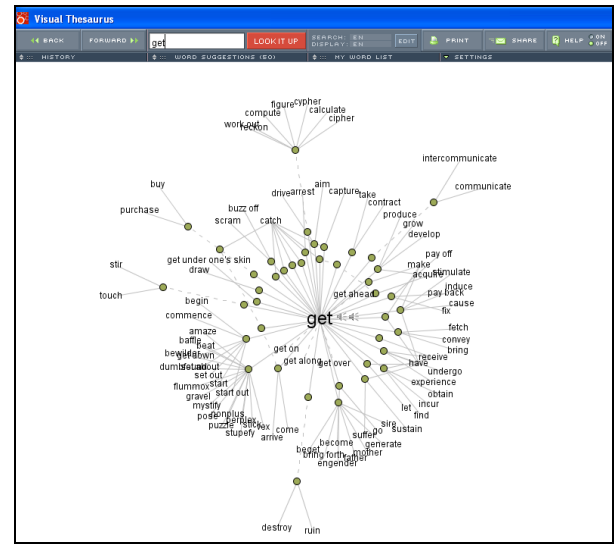

Fig. 2. A screen shot of Visual Thesaurus ${ }^{\circledR}$ with the word "get" at the center

\subsection{Reconfigure: show me a different arrangement}

Reconfigure interaction techniques provide users with different perspectives onto the data set by changing the spatial arrangement of representations. One of the essential purposes of Infovis is to reveal hidden characteristics of data and the relationships between them. A good static representation often serves this purpose, but a single representation rarely provides sufficient perspectives. Thus, many Infovis tools incorporate Reconfigure interaction techniques that allow users to change the way data items are arranged or the alignment of data items in order to provide different perspectives on the data set.

The sorting and rearranging columns operations in TableLens [33] are good examples of Reconfigure techniques. As shown in Fig. 3 , by sorting the "Horsepower" column, users can determine that horsepower values of vehicles are roughly correlated with cylinders, displacement, and weight. Also, users can rearrange the columns to compare attributes of interest side by side. Sorting and rearranging columns (or rows) features can be found in other Infovis systems containing tabular views as well, such as InfoZoom [40].

The capability of changing the attributes presented on the axes in a scatter plot view of Spotfire [2] is a similar, but different example of a Reconfigure technique. Changing the attributes assigned to $\mathrm{x}$ - and y-axes changes the sets of attributes or variables to be examined among the entire data set, so it eventually changes relationships between data items and provides different perspectives.

The baseline adjustment feature in a stacked histogram, as shown in Fig. 4, enables users to better compare the heights of subsections of the histogram [15]. Without this technique, it is difficult to compare the values of subsections (values of the West variable in the figure) not initially on the bottom of the histogram. The Selective Dynamic Manipulation (SDM) system [14], which introduced many interaction techniques for $2 \mathrm{D}$ and $3 \mathrm{D}$ visualization, provides a similar technique to compare the heights of bars in three-dimensional visualization. Since a distant object appears smaller than a nearby object in a 3D view, comparing the two objects' heights, for example, is challenging. SDM allows users to bring objects in a $3 \mathrm{D}$ view to a front $2 \mathrm{D}$ plane with a common baseline so that users can compare the sizes more accurately.

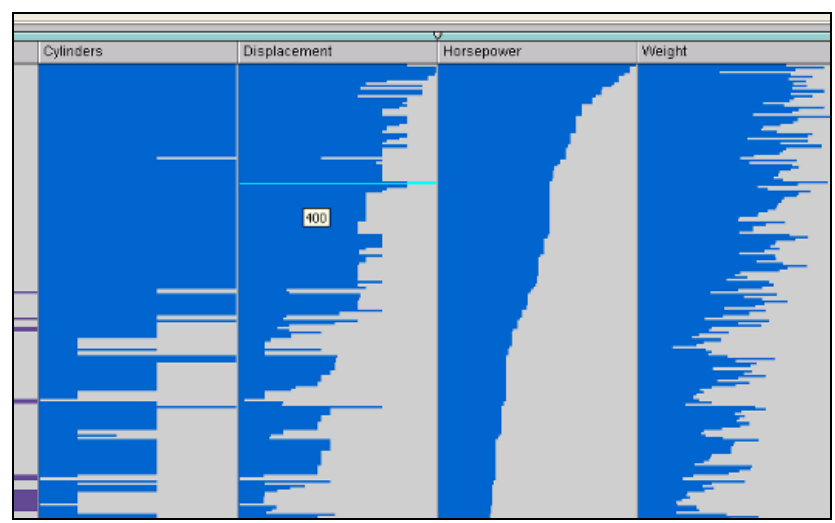

Fig. 3. A screen shot of TableLens using the sort function on the "Horsepower" column

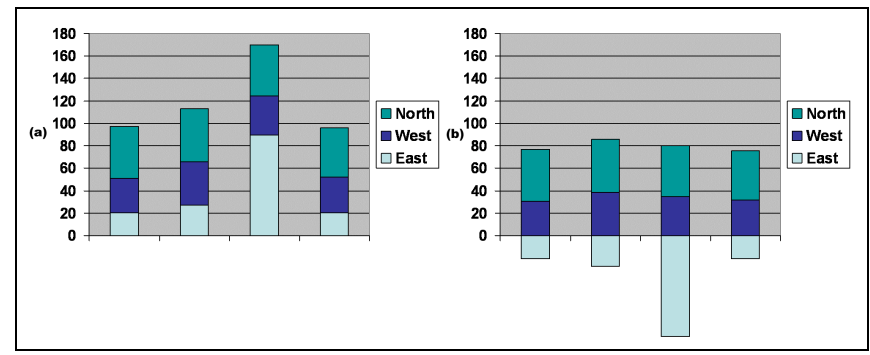

Fig. 4. Stacked histograms: (a) an original view and (b) a view with baseline adjustment

Other system's interaction techniques allow users to move data items more freely to make the arrangement more suitable for their mental model. For example, users of the online social network visualization system Vizster [21] can move nodes freely and thus can arbitrarily cluster a certain set of people (e.g., family, friends, and business contacts). The Data Mountain [34] that presents web browser favorites as thumbnails on an inclined plane is another similar example as it allows users to arrange groups of related web pages at various positions on the plane.

Reconfigure techniques also include a set of interaction techniques reducing occlusions. Since many Infovis systems present large amounts of data, individual data cases often visually overlap. Especially in 3D representation techniques, distant data items are often occluded by nearby data items in the same line of sight.

For example, the view rotation operation in many 3D Infovis systems (e.g., SDM [14]) helps reduce occlusion in a 3D visualization. Such a feature helps users rotate their line of sight to see through a cloud of data items. A similar, but slightly different example is a technique in ConeTrees [35] where users rotate a portion of the tree instead of rotating the line of sight in order to see occluded data items. 
Another example interaction in this category is the jitter operation as implemented in systems like Spotfire [2]. When many data cases are drawn to particular vertical or horizontal rows, items may overlap resulting in occlusion. By applying jitter, the position of each item is randomly shifted by a small spatial increment, thus uncovering many more items and providing a better sense of the density of items in a region. Fig. 5 illustrates the results of jitter in Spotfire. A similar technique in Dust \& Magnet is the "Spread Dust" operation that makes data items (dust particles) gradually repel each other so that occlusion decreases [55].

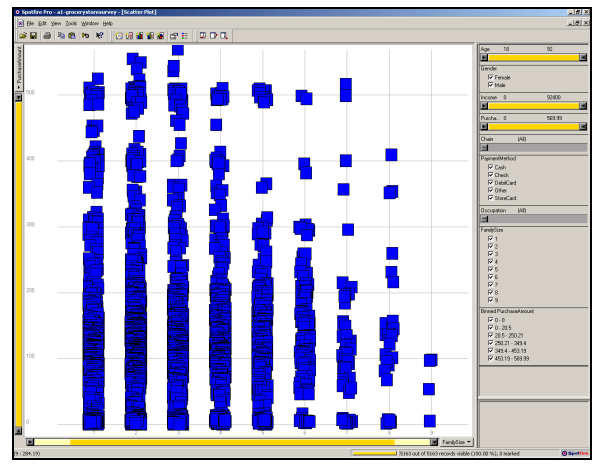

Fig. 5. A screen shot of Spotfire showing the result of the jitter technique.

\subsection{Encode: show me a different representation}

Encode techniques enable users to alter the fundamental visual representation of the data including visual appearance (e.g., color, size, and shape) of each data element. In Infovis systems, visual elements serve an important role not only because they can affect pre-attentive cognition but also because they are directly related to how users understand relationships and distributions of the data items. For instance, by encoding height information to a map using a spectrum of color, users can better identify the height information (e.g., the height of a mountain) without altering the spatial arrangement of the map.

Simply changing how the data is represented (e.g., changing a pie chart to a histogram) is an example of Encode. By changing a type of representation, users expect to uncover new aspects of relationship. Infovis systems that provide multiple representations of data, for example, Spotfire [2] and Xmdv tool [49], have this capability.

Another widely used technique of Encode is the set of interaction techniques that alter the color encoding of a data set. Many Infovis techniques (e.g., Dust \& Magnet [55], InfoScope by Macrofocus [27], and Spotfire [2]) enable users to adjust a color or a spectrum of colors for a certain variable. Since color encoding is changed instantly and dynamically, users can experiment with various color encoding schemes to find the most suitable one. Additive color encoding in Attribute Explorer [39] is an advanced color encoding technique, which helps users understand distributions of multiple variables rather than a single variable.

Beyond color encoding, many systems provide other encoding techniques, such as size (e.g., Dust \& Magnet [55]), orientation (e.g., Polaris [43]), font (e.g., SemaSpace [31]), and shape (e.g., Spotfire [2]). Since some of encoding techniques can be used simultaneously, they are often used together to encode many variables into representation. Again, interactivity is essential to help users find a proper encoding scheme.

\subsection{Abstract/Elaborate: show me more or less detail}

Abstract/Elaborate interaction techniques provide users with the ability to adjust the level of abstraction of a data representation. These types of interactions allow users to alter the representation from an overview down to details of individual data cases and often many levels in-between. The user's intent correspondingly varies between seeking more of a broad, contextual view of the data to examining the individual attributes of a data case or cases.

An exemplary interaction technique in this category is any technique from the set of details-on-demand operations. For example, the drill-down operation in a treemap visualization, such as SequoiaView (formerly known Cushion Tree [48]), allows a user to examine a particular sub-tree within an information hierarchy. Similarly, the animated details-on-demand techniques of SunBurst [42] (i.e., angular detail, detail inside, and detail outside) provide very similar functionality by allowing particular sub-trees in a hierarchy to be examined more closely without losing context of the entire structure. TableLens [33] also allows users to focus on a data case and its details (text of actual values) emerge. Furthermore, simple tool-tip interaction techniques that provide detailed information when a mouse cursor hovers over a data item also belong to this category: for example, SeeIT [1] as shown in Fig. 6 .

Another very common but slightly complex example of Abstract/Elaborate techniques is zooming (or geometric zooming if it is to be distinguished from semantic zooming). Through zooming, users can simply change the scale of a representation so that they can see an overview of a larger data set (using zoom-out) or the detailed view of a smaller data set (using zoom-in). A key point here is that the representation is not fundamentally altered during zooming. Details simply come more clearly into focus or fade away into context.

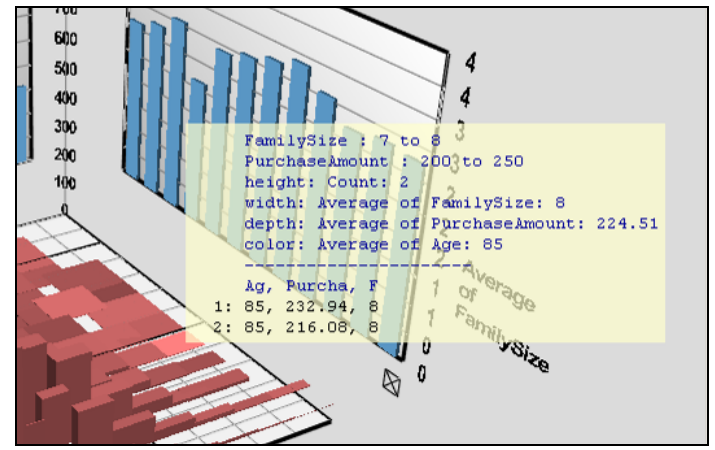

Fig. 6. A screen shot of SeelT showing the tool tip feature

\subsection{Filter: show me something conditionally}

Filter interaction techniques enable users to change the set of data items being presented based on some specific conditions. In this type of interaction, users specify a range or condition, so that only data items meeting those criteria are presented. Data items outside of the range or not satisfying the condition are hidden from the display or shown differently, but the actual data usually remain unchanged so that whenever users reset the criteria, the hidden or differently shown data items can be recovered. The user is not changing perspective on the data, just specifying conditions on which data are shown.

Dynamic query controls [3] as used in many Infovis systems (e.g., Spotfire [2]) are a representative example of this type of interaction. Users select ranges by moving sliders or particular values by clicking on check boxes and the data cases meeting those constraints are immediately shown. This type of interaction helps make a system feel much more responsive and live as compared to traditional batchoriented text queries. Variants of dynamic query controls such as alphasliders, rangesliders, and toggle buttons are used to filter textual data, numerical data, and categorical data, respectively.

The Attribute Explorer [39] extends dynamic query capabilities by changing the colors of filtered data items rather than removing them from the display, as shown in Fig. 7. This helps users understand the context of the dataset by showing nearby data items not quite meeting the filtering criteria.

The Name Voyager [53], a website that illustrates the popularity of baby names over time, also supports a filtering interaction. Instead of using specific controls, users can filter the data items (e.g., names) through keyboard interaction. For example, as shown in Fig. 8, when 
a user types "K", only baby names starting with $\mathrm{K}$ are shown on the display. If the user types "I" following K, the system filters the data set and only shows names that start with "KI". By this simple and intuitive interaction technique, Name Voyager provides a very natural visual exploration of the data. QuerySketch [52] is yet another interesting example of Filter techniques. QuerySketch allows users to draw a line graph freehand, and then the system retrieves and presents data cases with similar graphs. These graphs frequently represent time series data.

(a)

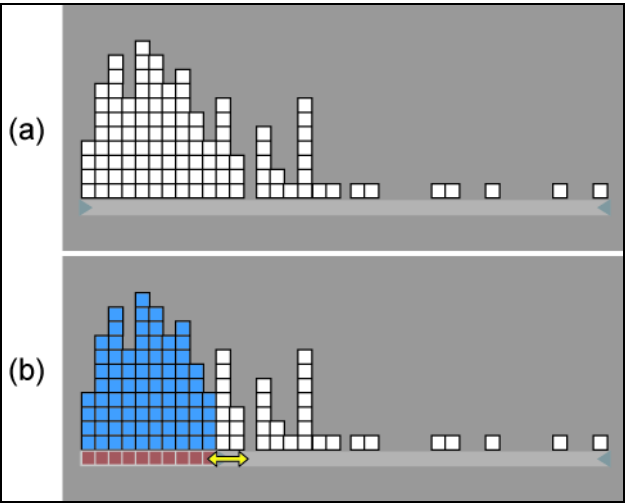

Fig. 7. Attribute Explorer style display: (a) before changing limits and (b) after changing the lower limit

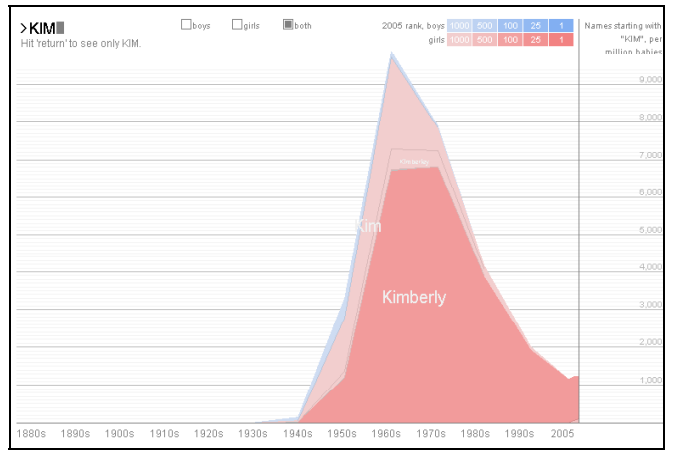

Fig. 8. A screen shot of Name Voyager showing names with "KIM"

\subsection{Connect: show me related items}

Connect refers to interaction techniques that are used to (1) highlight associations and relationships between data items that are already represented and (2) show hidden data items that are relevant to a specified item.

When multiple views are used to show different representations of the same data set (e.g., 3D scatter plot and 2D scatter plot as shown in Fig. 9), it may be difficult to identify the corresponding item for a data case in other view(s). To alleviate this difficulty, the brushing technique is used to highlight the representation of a selected data item in the other views being displayed. In Fig. 9, when a user selects a data item in the left view, the same data item of the right view is highlighted (circled in this case) simultaneously.

Connect interactions can apply to situations involving a single view as well. For example, in Vizster [21], hovering a mouse cursor over a node highlights directly connected nodes (friends) or neighbors of directly connected nodes (friends of friends). Here, the connection is not to other representations of the same item as in brushing but to items that harbor relationship to a focus element.

Connect interaction techniques also reveal related data items which are originally not shown. In Vizster, double clicking a node causes expansion of the node, so that the related nodes for the focus node (the person) are added. A similar but different example is the aforementioned Visual Thesaurus ${ }^{\circledR}$ [44], where clicking a word in a view reveals related words, and other unrelated words in the original view disappear. Keen readers might notice that this interaction technique was already categorized as Explore, which will be discussed more in the Discussion section.

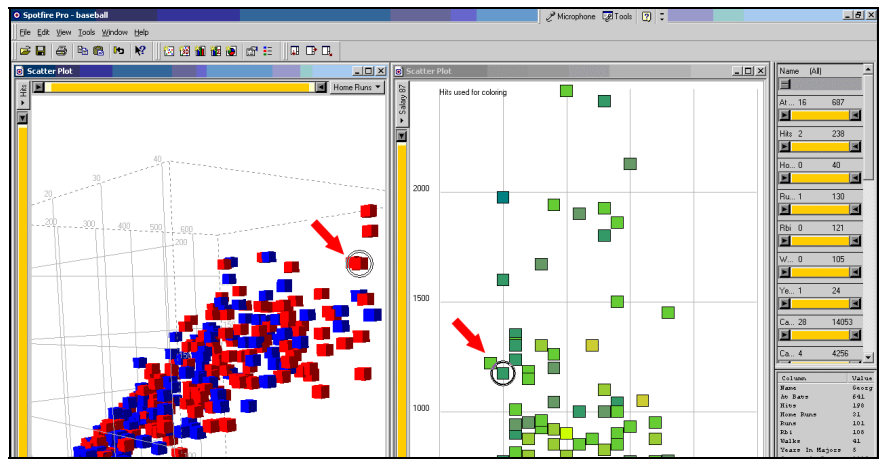

Fig. 9. A screen shot of Spotfire showing a brushing technique

\subsection{Other Interaction Techniques}

Other interaction techniques in Infovis systems certainly exist. For instance, consider a broad set of operations found commonly in many interactive applications. A few examples are listed below:

- Undo/redo: techniques that allow users to go backward or forward to pre-existing system states (e.g., undo, redo, history, and reset)

- Change configuration: techniques that allow users to change various configurations and settings of a system (e.g., change locations of dynamic queries in Spotfire [2])

Because these operations are common to many different types of applications and are not unique to Infovis, we have chosen not to include them in our scheme. This, however, does not diminish their value as useful interactive capabilities in information visualization.

\section{Discussion}

It is difficult to create categories of interaction techniques that are clear and comprehensive. The categories we proposed are based on our own perspective on interaction in Infovis and, thus, inherently debatable. In this section, we discuss issues with our categorization and the nature of interaction in Infovis.

Through the categorization process, we realized that the categories are not collectively exhaustive. Some techniques are difficult to classify and do not quite fit into any one of the categories. For example, as shown in Fig. 10, the water level technique in SeeIT [33], which visualizes multivariate data in a $3 \mathrm{D}$ view with projection walls, provides a movable baseline that can be adjusted up and down, so that users can compare the heights of 3D histograms. Here, users do not interact directly with data items and the representation of data items remains unchanged. It is a technique that adds a layer on top of the representation and plays a role as a cognitive aid to augment a user's ability to compare the values. This interaction simply did not seem to fit well into any of our categories.

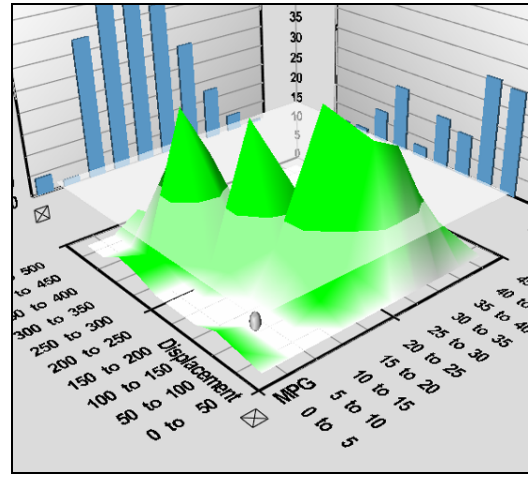

Fig. 10. A screen shot of SeelT showing the water level feature 
Some other interaction techniques appear to fulfill multiple user intents, which make it possible to classify them into several categories. For example, semantic zooming [32] is not only an Elaborate/Abstract technique due to its zooming capability, but also an Encode technique since the data representation can change as the zooming scale changes. The Magic Lens [17], which provides a different sub view on top of the main view using a lens metaphor, is another good example of having multiple intents. One popular usage of Magic Lens is using it as movable and stackable filter. However, it can also provide many other functions (e.g., color encoding, changing representation, zooming, and providing details), which makes it difficult to classify into a single category. The aforementioned interaction technique in Visual Thesaurus ${ }^{\circledR}[44]$ is another example, which is categorized as both Connect and Explore since users can explore new words by clicking a connected word.

We also considered other categories to be added to our final set. For example, we debated adding a Compare category but ultimately omitted it because we believe that Compare is a higher-level user goal or objective than the other user intents we identify. Compare simply can mean so many different things. In particular, the intents we identify often make up components of a broader comparison goal-"Let me Filter data so that I can compare items of interest"; "Let me Reconfigure to compare these two subsets more easily"; and "Let me Encode variable A to easily compare this attribute."

Despite all these debatable issues and exceptions, we still believe that this categorization, based on user intents, is a useful approach and has several strengths. In order to assess its utility in a systematic manner, we use Beaudouin-Lafon's three dimensions (i.e., descriptive, evaluative, and generative power) for evaluating interaction models. First, we believe that our user-intent-centric categorization has fairly good descriptive power in that it captures the characteristics of interaction techniques at a higher level. While a simple enumeration of interaction techniques often fails to embrace variants of existing techniques or new techniques, our categories are less vulnerable to new developments as long as they serve one of user intentions we have identified. Second, our categories can help designers and developers to examine whether users' needs are fulfilled by a system, which implies that it has an evaluative power to some degree. Understanding what is missing in a system in terms of supporting user intents could be a more meaningful way to evaluate the system than simply checking whether the system has a particular feature that is commonly used in other systems. Finally, we argue that it also has generative power. Even though the categorization may not directly help Infovis designers generate new ideas of interaction techniques, it provides at least some common vocabularies to think about different users' intentions when developing new techniques. Since understanding what users need promotes the creativity of designers [26], we believe that our categories may have contributions in that aspect.

We began this article with a statement that Infovis systems appear to have two different fundamental components: representation and interaction. Through our work and analysis, however, we came to a conclusion that maybe it is not so easy to separate the two. On the one hand, some may argue that interaction is all about representation in that it plays a role merely as an operator that changes representation. Without interaction, however, representation is no more than a static image. By supporting further exploration of data items, interaction enables users to have multiple perspectives and gain insight on the data set. It is what separates an Infovis system from a static image. We conclude that these two components are in a symbiotic relationship.

One way to distinguish representation and interaction might be through temporal characteristics. While representation is not dependent on time per se, interaction fundamentally involves changes over time. A basic tradeoff exists between the time to perform interaction activities (e.g., generating a different view) and the space required to present multiple static images (e.g., screen real estate). To achieve the same variety of representations without interaction, one would need a huge display. Thus, as can be seen, there is a tradeoff between using multiple static representations and one, interactive representation.

Nonetheless, the value of representation and interaction in helping users understand information, while reducing cognitive burden, makes it impossible to separate the two. More research is needed to better understand how to leverage each component to build optimal Infovis systems.

\section{Conclusions}

In this paper, we proposed seven different categories of interaction techniques based on user intents. We believe that this article makes two main contributions to the Infovis domain.

First, our efforts draw attention to the importance of interaction in Infovis research and reveal its subtle complexity. While existing research in the area often focuses on representation, we highlight the overshadowed, but very important interaction component and strongly argue that it provides a way to overcome the limits of representation and augment a user's cognition.

Second, we provide a novel user intent-based categorization to discuss and characterize interaction techniques in Infovis. In conjunction with other interaction taxonomies, our categories might be able to provide a bigger picture view of interaction. For example, using these categories, it would be useful and meaningful to discuss what type of user intent a system supports (or not) as well as what tasks a system supports (or not).

Certainly, simply having these categories is far from our eventual goal of establishing the science of interaction in Infovis. However, we believe that these categories are an initial step toward this direction. We believe that this categorization better articulates the ways in which interaction techniques are used, while providing a more useful common vocabulary (of user intent) for further discussion and application in the development of Infovis systems. Our categorization, coupled with an exhaustive list of interaction techniques as well as higher-level user tasks, would provide a holistic framework that moves closer to providing a true science of interaction.

\section{ACKNOWLEDGEMENTS}

This research is supported in part by the National Science Foundation via Award IIS-0414667 and the National Visualization and Analytics Center (NVAC ${ }^{\mathrm{TM}}$ ), a U.S. Department of Homeland Security Program, under the auspices of the SouthEast Regional Visualization and Analytics Center. It is also supported in part by GVU Center Seed Grant. The authors also wish to thank Erin Kinzel and Kevin Moloney for their careful review of this document.

\section{REFERENCES}

[1] ADVISOR Solutions Inc., "SeeIT," http://www.advizorsolutions.com/. 2007.

[2] C. Ahlberg, "Spotfire: an information exploration environment," SIGMOD Record, vol. 25, pp. 25-29, 1996.

[3] C. Ahlberg, C. Williamson, and B. Shneiderman, "Dynamic Queries for Information Exploration: An Implementation and Evaluation," presented at Conference on Human Factors in Computing Systems (CHI '92), Monterey, CA, USA, pp. 619-626, 1992.

[4] R. Amar, J. Eagan, and J. T. Stasko, "Low-Level Components of Analytic Activity in Information Visualization," presented at IEEE Symposium on Information Visualization (InfoVis '05), pp. 111-117, 2005.

[5] M. Beaudouin-Lafon, "Designing interaction, not interfaces," presented at the working conference on Advanced visual interfaces (AVI '04), Gallipoli (LE), Italy, pp. 15-22, 2004.

[6] R. A. Becker, W. S. Cleveland, and A. R. Wilks, "Dynamic Graphics for Data Analysis," Statistical Science, vol. 2, pp. 355-383, 1987.

[7] B. B. Bederson, J. Grosjean, and J. Meyer, "Toolkit design for interactive structured graphics," IEEE Transactions on Software Engineering, vol. 30, pp. 535-546, 2004. 
[8] J. Bertin, Semiology of graphics: University of Wisconsin Press, 1983.

[9] A. Buja, D. Cook, and D. F. Swayne, "Interactive High-Dimensional Data Visualization," Journal of Computational and Graphical Statistics, vol. 5, pp. 78-99, 1996.

[10] A. Buja, J. A. McDonald, J. Michalak, and W. Stuetzle, "Interactive data visualization using focusing and linking," presented at IEEE Conference on Visualization (Visualization '91), San Diego, California, pp. 156-163, 1991.

[11] S. K. Card, P. Pirolli, and J. D. Mackinlay, "The cost-of-knowledge characteristic function: display evaluation for direct-walk dynamic information visualizations," ACM Press New York, NY, USA, 1994, pp. 238-244.

[12] M. C. Chuah and S. F. Roth, "On the Semantics of Interactive Visualizations," presented at IEEE Symposium on Information Visualization (InfoVis '96), San Francisco, CA, USA, pp. 29-36, 1996

[13] M. C. Chuah and S. F. Roth, "On the Semantics of Interactive Visualizations," presented at IEEE Symposium on Information Visualization, San Francisco, CA, pp. 29-36, 1996.

[14] M. C. Chuah, S. F. Roth, J. Mattis, and J. Kolojejchick, "SDM: selective dynamic manipulation of visualizations," presented at ACM symposium on User interface and software technology (UIST '95), pp. 61-70, 1995.

[15] A. Dix and G. Ellis, "Starting simple: adding value to static visualisation through simple interaction," presented at the working conference on Advanced visual interfaces (AVI '98), L'Aquila, Italy, pp. 124-134, 1998.

[16] A. Dix, J. Finlay, G. D. Abowd, and R. Beale, Human-computer interaction, 3rd ed: Pearson Prentice Hall, 2004

[17] K. Fishkin and M. C. Stone, "Enhanced dynamic queries via movable filters," presented at Conference on Human Factors in Computing Systems (CHI '95), Denver, CO, USA, pp. 415-420, 1995.

[18] J. D. Foley, A. van Dam, S. K. Feiner, and J. F. Hughes, Computer Graphics: Principles and Practice in C, 2nd ed: Addison-Wesley Professional, 1995.

[19] G. W. Furnas, "Generalized fisheye views," presented at Conference on Human Factors in Computing Systems (CHI '86), Boston, MA, USA, pp. 16-23, 1986.

[20] Google Inc., "Google Earth," http://earth.google.com/.

[21] J. Heer and D. Boyd, "Vizster: Visualizing Online Social Networks," presented at IEEE Symposium on Information Visualization (InfoVis '05), Minneapolis, MN, USA, pp. 33-40, 2005.

[22] HumanIT, "InfoZoom," http://www.infozoom.com/enu/index.htm.

[23] Inxight Software Inc., "Table Lens," http://www.inxight.com/products $/ \mathrm{sdks} / \mathrm{t} \mathrm{l} /$.

[24] D. A. Keim, "Information Visualization and Visual Data Mining," IEEE Transactions on Visualization and Computer Graphics, vol. 8, pp. 1-8, 2002.

[25] R. Kosara, H. Hauser, and D. Gresh, "An Interaction View on Information Visualization," presented at EUROGRAPHICS 2003 (EG '03), pp. 123-137, 2003.

[26] B. Lawson, How Designers Think: The Design Process Demystified, 3rd ed: Architectural Press, 1997.

[27] Macrofocus GmbH, "InfoScope," http://www.macrofocus.com/public/ products/infoscope.html.

[28] T. Miller and J. T. Stasko, "The InfoCanvas: information conveyance through personalized, expressive art," presented at Conference on Human Factors in Computing Systems (CHI '01), Seattle, WA, USA, pp. 305-306, 2001.

[29] D. A. Norman, Things that make us smart. Reading, MA, USA: Addison-Wesley Pub. Co., 1993.

[30] D. A. Norman, The design of everyday things: Basic Books, 2002.

[31] D. Offenhuber and G. Dirmoser, "SemaSpace - Semantic Networks as Memory Theatre," http://residence.aec.at/didi/FLweb/semaspace.pdf.

[32] K. Perlin and D. Fox, "Pad: an alternative approach to the computer interface," presented at Computer graphics and interactive techniques, pp. 57-64, 1993.

[33] R. Rao and S. K. Card, "The table lens: merging graphical and symbolic representations in an interactive focus + context visualization for tabular information," presented at Conference on Human Factors in Computing Systems (CHI '94), Boston, MA, USA, pp. 318 - 322, 1994
[34] G. Robertson, M. Czerwinski, K. Larson, D. C. Robbins, D. Thiel, and M. van Dantzich, "Data mountain: using spatial memory for document management," presented at ACM symposium on User interface software and technology (UIST '98), San Francisco, CA, USA, pp. 153$162,1998$.

[35] G. G. Robertson, J. D. Mackinlay, and S. K. Card, "Cone Trees: animated 3D visualizations of hierarchical information," presented at Conference on Human Factors in Computing Systems (CHI '91), New Orleans, LA, USA, pp. 189-194, 1991.

[36] B. Shneiderman, "Tree visualization with tree-maps: 2-d space-filling approach," ACM Transactions on Graphics, vol. 11, pp. 92-99, 1992.

[37] B. Shneiderman, "The eyes have it: a task by data type taxonomy for information visualizations," presented at IEEE Symposium on Visual Languages, 1996, Boulder, CO, USA, pp. 336-343, 1996.

[38] R. Spence, Information Visualization: Design for Interaction, 2nd ed: Prentice Hall, 2007

[39] R. Spence and L. Tweedie, "The Attribute Explorer: information synthesis via exploration," Interacting with Computers, vol. 11, pp. 137146, 1998.

[40] M. Spenke and C. Beilken, "InfoZoom - Analysing Formula One racing results with an interactive data mining and visualisation tool," presented at International Conference on Data Mining, Cambridge University, United Kingdom, pp. 455-64, 2000.

[41] Spotfire Inc., "Spotfire," http://www.spotfire.com/. 2007.

[42] J. T. Stasko and E. Zhang, "Focus+ Context Display and Navigation Techniques for Enhancing Radial, Space-Filling Hierarchy Visualizations," presented at IEEE Symposium on Information Visualization (InfoVis '00), Salt Lake City, UT, USA, pp. 57-65, 2000.

[43] C. Stolte, D. Tang, and P. Hanrahan, "Polaris: a system for query, analysis, and visualization of multidimensional relational databases," IEEE Transactions on Visualization and Computer Graphics, vol. 8, pp. 52-65, 2002.

[44] Thinkmap Inc., "Thinkmap Visual Thesaurus," http://www.visual thesaurus.com/.

[45] J. J. Thomas and K. A. Cook, "Illuminating the Path." Los Alamitos, CA, USA: IEEE, 2005.

[46] E. R. Tufte, Envisioning information: Graphics Press, 1990.

[47] L. Tweedie, "Characterizing Interactive Externalizations," presented at Conference on Human Factors in Computing Systems (CHI '97), Atlanta, GA, pp. 375 - 382, 1997.

[48] J. J. Van Wijk and H. Van de Wetering, "Cushion treemaps: visualization of hierarchical information," presented at IEEE Symposium on Information Visualization (InfoVis '99), San Francisco, CA, USA, pp. 73-78, 1999.

[49] M. O. Ward, "XmdvTool: integrating multiple methods for visualizing multivariate data," presented at IEEE Conference on Visualization (Visualization '94), Washington, DC, USA, pp. 326-333, 1994.

[50] M. O. Ward and J. Yang, "Interaction Spaces in Data and Information Visualization," presented at Joint Eurographics/IEEE TCVG Symposium on Visualization, Konstanz, Germany, pp. 137-145, 2004.

[51] C. Ware, Information Visualization: Perception for Design. San Diego, CA, USA: Academic Press, 2000.

[52] M. Wattenberg, "Sketching a graph to query a time-series database," presented at Conference on Human Factors in Computing Systems (CHI '01), Seattle, WA, USA, pp. 381-382, 2001.

[53] M. Wattenberg and J. Kriss, "Designing for Social Data Analysis," IEEE Transactions on Visualization and Computer Graphics, vol. 12, pp. 549-557, 2006.

[54] L. Wilkinson, The Grammar of Graphics, 2nd ed. New York, NY, USA: Springer, 2005.

[55] J. S. Yi, R. Melton, J. T. Stasko, and J. A. Jacko, "Dust \& Magnet: multivariate information visualization using a magnet metaphor," Information Visualization, vol. 4, pp. 239-256, 2005.

[56] M. X. Zhou and S. K. Feiner, "Visual task characterization for automated visual discourse synthesis," presented at Conference on Human Factors in Computing Systems (CHI '98), Los Angeles, CA, USA, pp. 392-399, 1998 